\title{
Delivery of a PCR amplified DNA fragment into cells: a model for using synthetic genes for gene therapy
}

\author{
S Li, M Brisson, Y He and L Huang \\ Laboratory of Drug Targeting, Department of Pharmacology, W1351 Biomedical Science Tower, University of Pittsburgh School of \\ Medicine, Pittsburgh, PA 15261, USA
}

\begin{abstract}
Synthetic genes offer many potential advantages over conventional plasmid DNA, such as simplicity in purification, absence of endotoxin contamination, and more importantly, flexibility in chemical modifications to render them specific properties. We have used PCR amplified fragments as a model to test the feasibility of using synthetic genes for gene therapy. The CAT reporter gene driven by the CMV promoter (CMV-CAT), ie a nuclear expression system, or by the bacteriophage T7 promoter (T7-CAT), ie a cytoplasmic expression system, was used to evaluate this concept. The expression efficiency of both plasmids ( $p U C C M V-C A T$ and $p T 7-C A T$ ) and their
\end{abstract}

corresponding PCR fragments (fCMV-CAT and fT7-CAT) were compared on a molar basis. Limited expression of CAT was found with fCMV-CAT. However, fT7-CAT consistently gave a CAT activity comparable to that of pT7CAT. When fT7-CAT was codelivered with $p C M V / T 7-T 7 p o l$ (a self-amplifying T7 RNA polymerase autogene), high CAT activity could be detected up to 9 days. This expression was much longer than the duration of expression with a nuclear expression system. These encouraging results imply that gene therapy with synthetic genes could be both feasible and efficient.

Keywords: gene therapy; synthetic genes; polymerase chain reaction; liposome

\section{Introduction}

Gene therapy has become one of the fastest developing fields of biomedicine in recent years. ${ }^{1}$ Successful gene transfer depends on the use of a proper vehicle, or vector, which ideally should be highly efficient but not immunogenic or cytotoxic. Accordingly, much effort has recently been devoted to the improvement of delivery vehicles. ${ }^{2}$ Less attention, however, has been paid to the problems associated with the genes to be delivered. Plasmid DNA is purified from bacteria. Although much progress has been made in the purification process, the existing procedure is relatively cumbersome and not cost effective. Additionally, contamination by endotoxin remains problematic. ${ }^{3}$ Humans and most primates are highly sensitive to endotoxin. ${ }^{4,5}$ High doses can cause circulatory collapse in man; ${ }^{6}$ therefore, endotoxin contamination of plasmid DNA has become an important safety concern for human gene therapy. Furthermore, endotoxin can severely decrease the transgene expression in the transfected cells (unpublished data). Transfection activity of DNA may vary from batch to batch due to various degrees of endotoxin contamination (unpublished data). Therefore, production of DNA with reproducible purity and biological activity continues to be a major obstacle in efficient gene transfer. These problems might be solved by the use of synthetic genes, ie DNA synthesized chemically. There are additional advantages in using synthetic genes. First,

Correspondence: L Huang

The first two authors contributed equally to this study.

Received 3 May 1996; accepted 13 January 1997 purification of chemically synthesized DNA is well developed. More importantly, chemical modification can be manipulated according to specific needs. For example, stability of the gene could be improved by incorporating phosphorothioate to the ends of synthetic genes. Targeting ligands may also be linked to the synthetic genes for site-specific delivery. These potential advantages are not shared by conventional plasmid DNA. Eventually, gene therapy might be accomplished by using polydeoxynucleotides encoding a therapeutic gene. This study is designed to address the above issues by using PCR amplified, linear DNA fragments as a model for the double stranded DNA which is to be obtained by total synthesis. Data obtained thus far indicate that this approach is both feasible and significant.

\section{Results and discussion}

It has been shown previously that linear plasmid DNA microinjected into cultured mammalian cells gave a higher homologous recombination frequency than that of supercoiled molecules. ${ }^{7}$ However, microinjection is not practical in most cases for in vivo gene therapy and direct application of linear DNA for gene transfer has been unsuccessful. ${ }^{8}$ In this study, two different types of PCR amplified fragments, fCMV-CAT and fT7-CAT (Figure 1), were used to test the feasibility of using synthetic genes for gene therapy with liposomes as a delivery vehicle. They represented a nuclear gene expression system and a cytoplasmic gene expression system, respectively. A detailed description of the latter gene expression system can be found in Refs 9 and 10.9,10 Briefly, it is composed of a bacteriophage T7 promoter and its corresponding RNA 

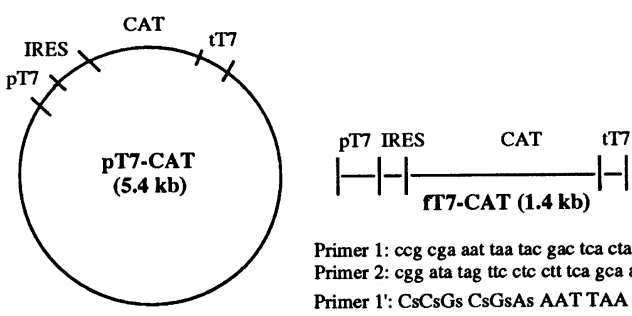

Primer 1: $\operatorname{ccg}$ cga aat taa tac gac tca cta t Primer 2: cgg ata tag ttc ctc ctt tca gca a

Primer 1': CsCsGs CsGsAs AAT TAA TAC GAC TCA CTA T Primer 2': CsGsGs AsTsA TAG TTC CTC CTT TCA GCA A

Cytoplasmic expression system
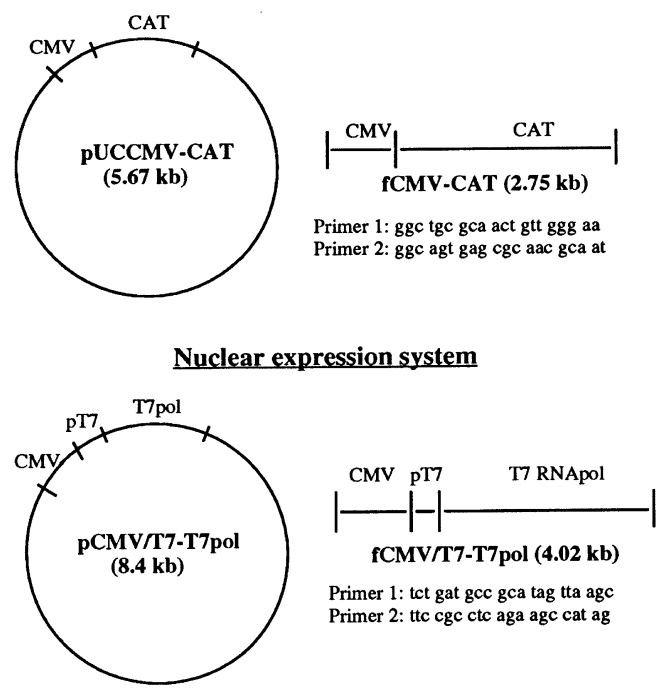

Dual expression system

Figure 1 Maps of the linear DNA fragments and of conventional plasmids. Sequences of primers used to generate the PCR fragments are also shown. pT7-CAT: T7-CAT plasmid; fT7-CAT: T7-CAT fragment; pUCCMV-CAT: $C M V$-CAT plasmid;fCMV-CAT: CMV-CAT fragment; pCMV/T7-T7pol: an autogene plasmid where the T7 RNA polymerase gene is under the control of both $C M V$ and T7 promoters; $C C M V / T 7-$ T7pol: CMV/T7-T7pol fragment; $p$ T7: T7 promoter; $t T 7$ : T7 terminator; T7pol: T7 RNA polymerase coding gene; IRES: internal ribosome entry site sequence of encephalomyocarditis virus.

polymerase and is independent of host endogenous transcriptional machinery. The T7 RNA polymerase remains primarily in the cytoplasm owing to its lack of a nuclear localization signal; ${ }^{11}$ therefore, nuclear transport of the plasmid DNA is not necessary. Rapid and transient expression of a CAT reporter gene, pT7-CAT, has been previously demonstrated following co-delivery with purified T7 RNA polymerase. ${ }^{9}$ When PT7-CAT was codelivered with an enzyme regeneration system, pT7-AUTO $2 \mathrm{C}^{-}$autogene, along with the purified enzyme, strong and sustained CAT expression was achieved, which was greater than that seen with pUCSV2CAT, a nuclear expression system. ${ }^{12}$ Both cytoplasmic gene expression system and nuclear gene expression system have been used to express molecules with pharmacological activity.

Transfection with 293-T7 cells

As an initial approach to test the idea of using synthetic genes, we compared the expression efficiency of plasmid DNA and linear DNA fragments delivered by DC-chol:DOPE liposomes or LPD. The efficiency of the cytoplas- mic expression system was also compared with that of the nuclear expression system. To make the comparison of the two systems straightforward, a cell line (293-T7) which produces endogenous T7 RNA polymerase was employed. The results are shown in Figure 2. Strong CAT gene expression was found in cells transfected with fT7CAT if delivered by DC-chol:DOPE liposomes. The level of expression was similar to that of pT7-CAT and also that of a nuclear expression plasmid pUCCMV-CAT delivered by DC-chol:DOPE liposomes. Very limited expression was obtained for fCMV-CAT delivered by DC-chol:DOPE liposomes or LPD complex when compared with pCMV-CAT on a molar basis. However, the CAT expression with fCMV-CAT was one third to approximately a half of that with pCMV-CAT when compared on a weight basis ( $1 \mu \mathrm{g}$ of DNA) (data not shown). There are many possible reasons for the lower expression of fCMV-CAT, one of which being its linear structure. Linear DNA complexed with cationic lipids or polymers might be less efficiently transported to the nucleus or transcribed in the nucleus. Figure 2 also demonstrates that CAT activity was less for pT7-CAT and further less for fT7-CAT if they were delivered by LPD rather than by DC-chol:DOPE liposomes. This might be due to overcondensation of DNA by polycations. Particularly, linear DNA may be more condensed due to the lack of structural constraints seen with supercoiled DNA. Highly condensed DNA, as fT7-CAT condensed by polylysine in the LPD complex, probably does not allow efficient un-coating of DNA in the cytoplasm for transcription. This was indirectly confirmed in an in vitro transcription assay (Figure 3). pT7-CAT showed little difference in its transcription before and after complexation with DC-chol:DOPE liposomes. However, transcription efficiency of fT7-CAT decreased greatly after its interaction with DCchol:DOPE liposomes. Complexation with polylysine

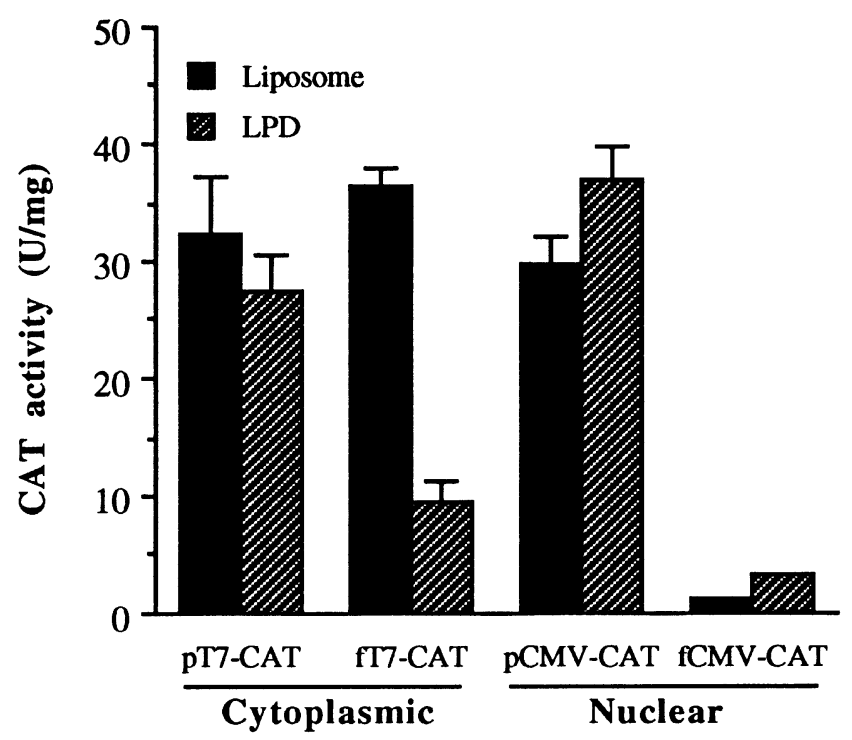

Figure 2 Comparison of the CAT gene expression from plasmid and linear DNA fragment delivered by DC-chol:DOPE liposomes or LPD. 293-T7, a 293 cell line stably transfected with $\mathrm{pCMV} / \mathrm{T7}-\mathrm{T} 7$ pol, produces endogenous T7 RNA polymerase. 0.27 pmol of DNA, complexed with liposomes or formulated in LPD complex, was used to transfect 293-T7 cells for 4 $h$. CAT assay was performed $48 \mathrm{~h}$ following the transfection. See Figure 1 for abbreviations of DNA. 


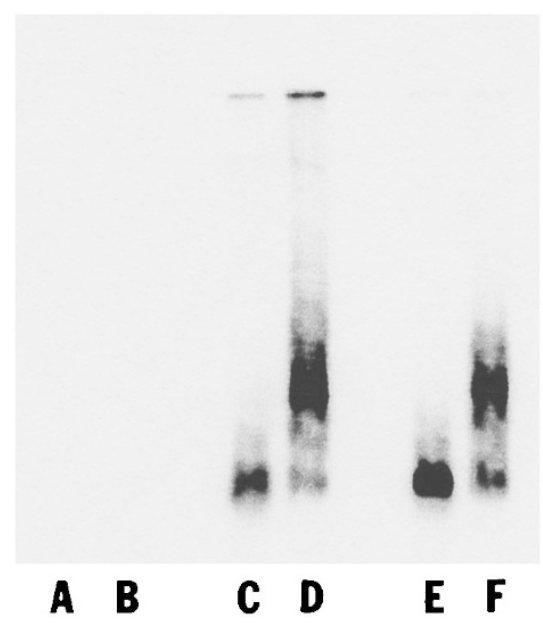

Figure 3 Comparison of the in vitro transcription of plasmid and linear DNA fragment. T7-CAT, alone or complexed with polycations was used as the template and transcribed RNA was analysed as described in Materials and methods. A, fT7-CAT/polylysine complex; B, pT7CAT/polylysine complex; C, fT7-CAT/DC-chol:DOPE complex; D, pT7CAT/DC-chol:DOPE complex; E, fT7-CAT alone; F, pT7-CAT alone.

completely inhibited the transcription of pT7-CAT and fT7-CAT by T7 RNA polymerase. It was suggested by a recent study ${ }^{13}$ that DNA and lipid must dissociate before transcription can occur in the nucleus. Data in this study imply that T7-CAT DNA does not necessarily have to dissociate from cationic lipids in the cytoplasm; however, favorable conformation of the complex is necessary for accessibility to T7 RNA polymerase. fT7-CAT differed from pT7-CAT in its requirements for a delivery vehicle. Rules for the optimization of delivery vehicles for the plasmid DNA may not necessarily apply to the linear DNA.

While transfection efficiency of linear DNA could be optimized for both the cytoplasmic expression system and nuclear expression system, data from the above experiment suggest that gene transfer with unmodified linear DNA works better with a cytoplasmic expression system, ie fT7-CAT rather than the nuclear expression system, ie fCMV-CAT. Therefore, the gene transfer potential of synthetic genes was further investigated by focusing on the cytoplasmic expression system.

Figure 4 shows the expression of the CAT reporter gene as a function of DNA concentration. The CAT activity increased with increasing amounts of T7-CAT, but reached a plateau when the T7-CAT was above 0.54 pmol. Failure in further increases of CAT activity with continuous increases in the amount of T7-CAT might be due to, among other factors, the amount of T7 RNA polymerase produced in 293-T7 cells. Because overexpression of T7 RNA polymerase is toxic to the cells, ${ }^{12}$ probably only those 293-T7 cells which produce moderate amounts of enzyme could survive the selection after cotransfection. No significant difference was found between pT7-CAT and fT7-CAT when they were delivered by liposomes.

Figure 5 demonstrates the expression of CAT reporter gene as a function of time. CAT expression was apparent at 1 day following the transfection. The CAT activity peaked at day 5 and declined slowly thereafter. The level of expression at day 9 was about a quarter of the peak

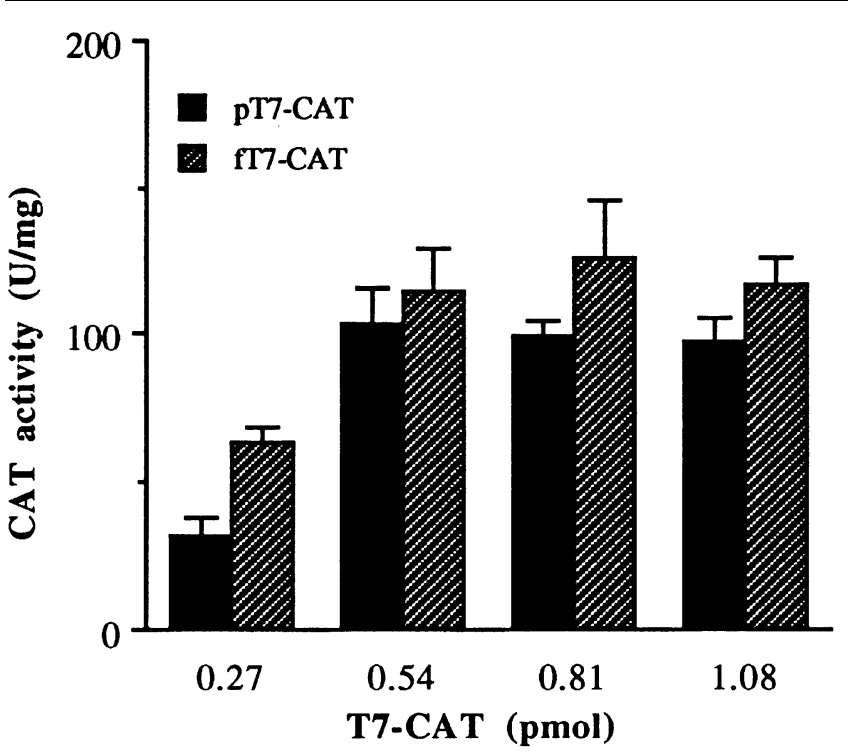

Figure 4 Expression of CAT reporter gene as a function of DNA concentration. 293-T7, a cell line stably transfected with $p C M V / T 7-T 7 p o l$, produces endogenous T7 RNA polymerase. Various amounts of DNA, complexed with DC-chol:DOPE liposomes at a ratio of $10 \mathrm{nmol} \mathrm{lipid/ \mu g}$ DNA, were used to transfect 293-T7 cells for $4 \mathrm{~h}$. CAT assay was performed $48 \mathrm{~h}$ following the transfection.

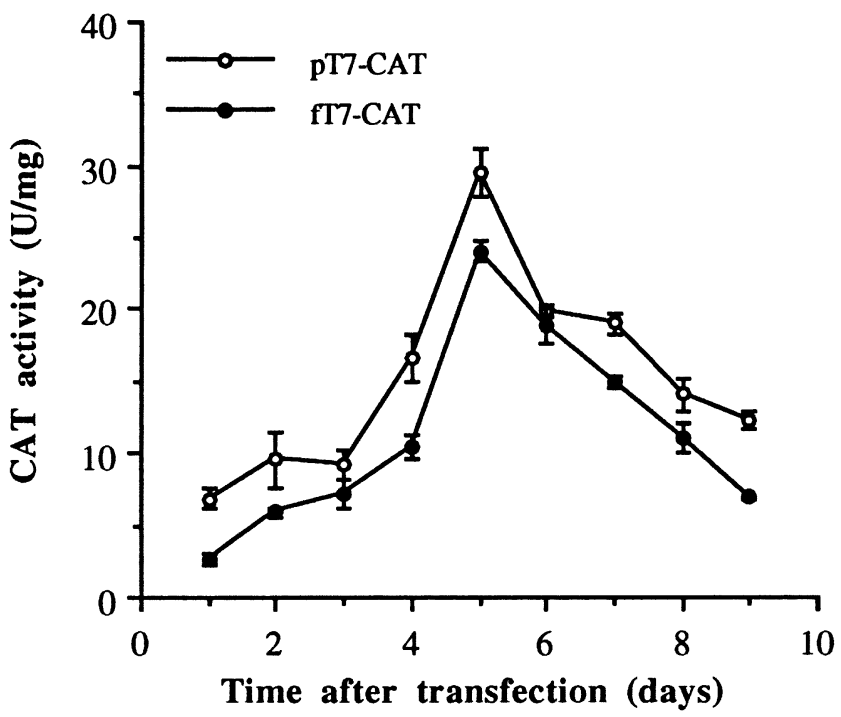

Figure 5 CAT expression as a function of time. T7-CAT was delivered to 293-T7 cells by DC-chol:DOPE liposomes as described in Figure 2. Cells were collected daily for 9 days. Cells were split 1:1 once on day 4 when they were $100 \%$ confluent.

level. This duration of CAT expression is longer than that seen with the nuclear expression system which is usually 3-5 days. ${ }^{12}$ This might be due to the fact that some of the T7-CAT remained associated with liposomes following release into the cytoplasm. This could prevent DNA from rapid degradation by cytoplasmic enzymes allowing for sustained gene expression. No significant difference in CAT activity was found between pT7-CAT and fT7-CAT, suggesting that linear DNA fragment delivered to the cells is not necessarily degraded more rapidly than plasmid DNA when cationic liposomes were used as a vector. It should be noted that a sharp increase in gene 
expression occurred following cell splitting. Such a phenomenon was also noticed in normal 293 cells (data not shown). Its mechanism is not clear at the moment. It is possible that cell splitting results in activation of some relevant cellular functions which are favorable for transgene expression.

\section{Transfection with 293 cells}

After demonstrating the feasibility of using fT7-CAT, the efficiency of this approach was then assessed using normal 293 cells. When T7-CAT was codelivered into 293 cells with purified T7 RNA polymerase, limited expression was observed for both plasmid and PCR fragments (data not shown). Toxicity was also noticed, which is in sharp contrast to the high CAT activity with minimal toxicity seen with 293-T7 cells. This might be due to exogenous T7 RNA polymerase being more toxic to cells than the enzyme produced endogenously. Thus, an autogene, pCMV/T7-T7pol (Figure 1), was employed in the subsequent studies.

pCMV/T7-T7pol is a dual expression system since the T7 RNA polymerase gene is under the control of both the CMV and T7 promoters. Following intracellular delivery, a small amount of the pCMV/T7-T7pol may be transported to the nucleus for the initial expression of T7 RNA polymerase. Once the enzyme is produced, the autogene could further amplify its expression via the T7 promoter and drive the synthesis of the transgene products in the cytoplasm.

As shown in Figure 6, higher expression of CAT was indeed obtained when the autogene was codelivered with T7-CAT. The expression of CAT activity increased steadily with increasing pCMV/T7-T7pol. The transfection efficiency of pT7-CAT was comparable to that of fT7CAT. No toxicity was noticed at any molar ratios examined if the cell confluence was above $70 \%$ at the time of transfection. However, at low cell confluency, toxicity was noticed if the molar ratio was above one-third. Further studies examining the duration of gene

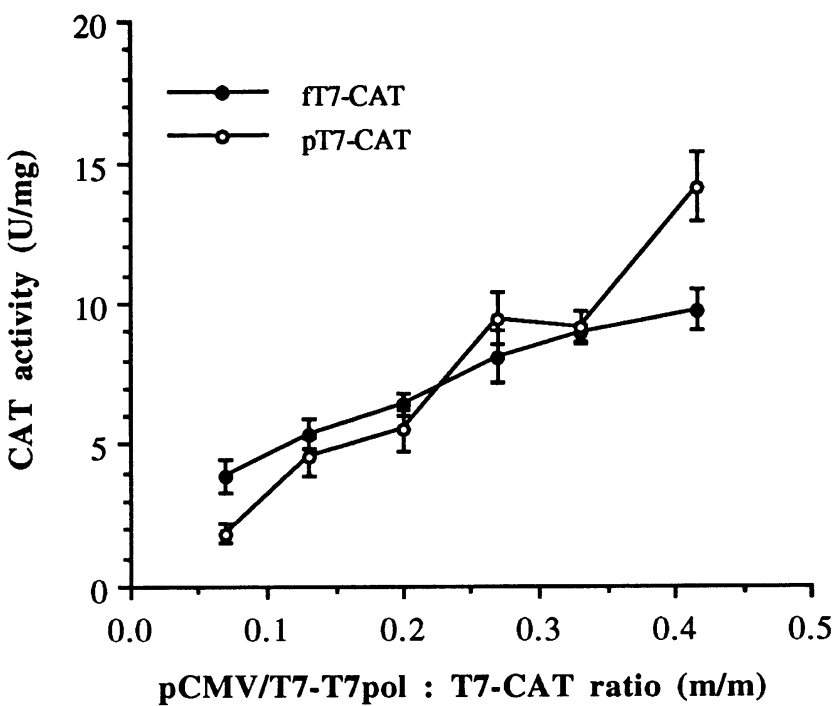

Figure 6 Co-delivery of T7-CAT with $p C M V / T 7-T 7 p o l$ autogene into 293 cells. A mixture of 0.27 pmol T7-CAT and varying amount of autogene,

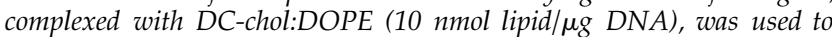
transfect 293 cells for $4 \mathrm{~h}$. CAT assay was performed $48 \mathrm{~h}$ following transfection.
Table 1 CAT reporter gene expression in 293 cells transfected with either plasmid DNA or PCR fragment ${ }^{\mathrm{a}}$

Type of DNA CAT activity $(\mathrm{U} / \mathrm{mg})$

pT7-CAT + pCMV/T7-T7pol

$3.24 \pm 0.78$

fT7-CAT + fCMV/T7-T 7pol

$6.08 \pm 1.23$

${ }^{a} \mathrm{~A}$ mixture of either two plasmids $(1 \mu \mathrm{g}$ pT7-CAT and $0.3 \mu \mathrm{g}$ pCMV/T7-T 7pol) or two PCR fragments $(1 \mu \mathrm{g}$ fT 7-CAT and $0.3 \mu \mathrm{g}$ fCMV/T7-T7pol) was complexed with DC-chol:DOPE liposomes (10 nmol lipid/ $\mu \mathrm{g}$ DNA). The complex was then used to transfect 293 cells for $4 \mathrm{~h}$. CAT activity was assayed $48 \mathrm{~h}$ following transfection.

expression on 293 cells showed a similar picture to that of 293-T7 cells (data not shown).

Table 1 suggests that pCMV/T7-T7pol could be replaced by fCMV/T7-T7pol. Codelivery of two PCR fragments into 293 cells resulted in a CAT gene expression two times higher than that of codelivery of plasmids when compared on a weight basis. Higher activity with fragments might be due to the fact that more molecules of both the target gene and autogene were delivered to the cells owing to the relatively smaller size of PCR fragments.

Last and more important, is the finding that a PCR fragment containing phosphorothioate linkage was essentially as active as the normal PCR fragment (Table 2). Phosphorothioate modification has been used solely in synthetic oligodeoxynucleotides to improve their stability. Therefore, inclusion of phosphorothioate linkages in the linear PCR fragment would mimick a synthetic gene. Though the phosphorothioate linkages were too few to see a clear beneficial effect, this study did suggest that gene therapy using a chemically modified synthetic gene is feasible.

We are aware of the fact that PCR fragment could be contaminated with endotoxin since Taq polymerase comes from bacteria. It has been shown that endotoxin can severely decrease the transgene expression in the transfected cells. Therefore, the efficiency of a synthetic gene approach might be underestimated by using a PCR fragment as a model. With synthetic genes, endotoxin would not be a problem. Also, synthetic genes are not

Table 2 CAT reporter gene expression in 293-T7 cells transfected with fT7-CAT with and without phosphorothioate linkages ${ }^{\mathrm{a}}$

\begin{tabular}{lcc}
\hline & \multicolumn{2}{c}{ CAT activity $(\mathrm{U} / \mathrm{mg})$} \\
\cline { 2 - 3 } & 0.27 pmol & $0.54 \mathrm{pmol}$ \\
\hline fT7-CAT with PS linkages & $34.56 \pm 5.23$ & $76.89 \pm 10.96$ \\
fT7-CAT without PS linkages & $26.76 \pm 4.73$ & $67.89 \pm 12.34$
\end{tabular}

afT7-CAT with or without phosphorothioate linkages at indicated concentrations was complexed with DC-chol:DOPE liposomes (10 nmol lipid/ $\mu \mathrm{g}$ DNA) and then used to transfect 293T7 cells for $4 \mathrm{~h}$

CAT activity was assayed $48 \mathrm{~h}$ following transfection. PS: phosphorothioate. 
associated with the problem of mutation which could occur in PCR. ${ }^{14}$ The existing problem with synthetic genes would be its high cost. However, considering the rapid reduction in the cost of antisense oligodeoxynucleotides, the problem of cost for synthetic genes might be solved in the near future.

In summary, we have demonstrated with 293 cells that transfection with linear DNA is both feasible and efficient. Preliminary studies showed that other types of cell lines could also be efficiently transfected with linear DNA given a suitable delivery vehicle. This makes gene therapy with synthetic genes a more practical approach worthy of further exploration.

\section{Materials and methods}

\section{Materials}

T7 RNA polymerase (New England Biolabs, Beverly, MA, USA) was used without further purification. All the chemicals for PCR including Taq polymerase, nucleotides and buffer were purchased from Gibco BRL (Gaithersburg, MD, USA). Acetyl-coenzyme A, chloramphenicol, Triton X-100 were from Sigma (St Louis, MO, USA). ${ }^{3} \mathrm{H}$ acetyl-coenzyme $\mathrm{A}$ and Beta-Max scintillation cocktail were from ICN Biomedicals (Costa Mesa, CA, USA). Dioleoylphosphatidylethanolamine (DOPE) and cholesteryl-hemisuccinate (CHEMS) were obtained from Avanti Polar Lipids (Birmingham, AL, USA). $3 \beta[N-$ ( $N^{\prime}, N^{\prime}$-dimethylaminoethane)-carbamoyl] cholesterol (DC-chol) was synthesized according to Gao and Huang. ${ }^{15}$ Unilamellar small liposomes of approximately $150 \mathrm{~nm}$ in diameter were prepared by microfluidization of a hydrated mixture of DC-chol and DOPE $(3: 2, \mathrm{~m} / \mathrm{m})$, and filter sterilized. $\mathrm{pH}$-sensitive liposomes composed of CHEMS and DOPE $(3: 2, \mathrm{~m} / \mathrm{m})$ were prepared by sonication.

\section{Plasmid DNA and PCR amplified linear fragments}

pT7-CAT which is the same as pT7-EMC-CAT, an expression plasmid of the CAT gene under the control of the bacteriophage T7 promoter ${ }^{16}$ was a kind gift from Dr B Moss (NIH, Bethesda, MD, USA). Plasmid pUCCMVCAT was constructed in this laboratory by Dr H Farhood. ${ }^{17}$ pCMV/T7-T7pol, an autogene composed of the T7 RNA polymerase gene under the control of both CMV and T7 promoters, was constructed in this laboratory by Dr JP Yang. A HindIII-BamHI fragment from pAR3126 ${ }^{11}$ containing the cDNA of T7 RNA polymerase was inserted into the corresponding sites of pcDNA3 plasmid vector (Invitrogen, San Diego, CA, USA) to generate pCMV/T7-T7pol. Detailed description of this autogene will be published elsewhere. The maps of the plasmids and the linear PCR amplified fragments are shown in Figure 1. All the plasmids were amplified in E. coli and purified by the $\mathrm{CsCl}$ gradient method. ${ }^{18}$ For PCR of pT7-CAT and pCMV-CAT, $10 \mathrm{ng}$ of plasmid template, 50 pmol of each primer (shown in Figure 1), 0.2 mM dNTPs, and 2.5 units of Thermus aquaticus (Taq) DNA polymerase were used. The PCR protocol consists of 4 min of denaturation at $94^{\circ} \mathrm{C}$, then 30 cycles of $45 \mathrm{~s}$ at $94^{\circ} \mathrm{C}, 1 \mathrm{~min}$ at $68^{\circ} \mathrm{C}$, and $2 \mathrm{~min}$ at $72^{\circ} \mathrm{C}$ and finally extension for $5 \mathrm{~min}$ at $72^{\circ} \mathrm{C}$. The resulting PCR amplified fragments were extracted with phenol-chloroform, ethanol-precipitated, resuspended in water and then further purified by passing thorough a size-exclusion filter. Similarly, fT7-CAT containing phosphorothioate linkage were amplified using two phosphodiester/phosphorothioate copolymers as primers (shown in Figure 1). For PCR of pCMV/T7-T7pol, a $50^{\circ} \mathrm{C}$ annealing temperature was used and specific fragment was further separated from other fragments by gel purification following the purification procedure as mentioned above.

\section{Preparation of $L P D$ complex}

LPD complex was prepared before transfection by mixing $\mathrm{pH}$-sensitive liposomes containing CHEMS and DOPE and the cationic DNA-polylysine (1:0.75, w/w) complex according to the method described by Lee and Huang. ${ }^{19}$ The lipid to DNA ratio in the final LPD complex was 5:1 $(w / w)$. The resulting LPD complex was cationic in charge. The molecular weight of polylysine was 25600 .

\section{Tissue culture}

Human embryonic kidney 293 cells, which were routinely tested and found to be free of Mycoplasma, were cultured in DMEM medium supplemented with $10 \%$ fetal bovine serum, penicillin (200 units $/ \mathrm{ml}$ ) and streptomycin $(100 \mu \mathrm{g} / \mathrm{ml})$ in a humidified atmosphere with $5 \% \mathrm{CO}_{2}$ at $37^{\circ} \mathrm{C}$.

293-T7, a 293 cell line which produces endogenous T7 RNA polymerase, was established by cotransfecting 293 cells with pCMV/T7-T7pol and pBK-CMV $\left(n e o^{\mathrm{r}}\right)$ phagemid and further selection by G418. The cells were cultured in DMEM medium supplemented with G418 (0.4 $\mathrm{mg} / \mathrm{ml})$.

\section{Transfection}

Cells (293 or 293-T7 cells) cultured in 24-well plates (about $70-80 \%$ confluent) were used for transfection. DNA (plasmid or PCR fragment), complexed with DCchol:DOPE liposomes (10 nmol lipid/ $\mu \mathrm{g}$ DNA) or formulated in a LPD complex, was used to transfect 293-T7 cells (0.27 pmol DNA per well). For 293 cells, pCMV /T7-T7pol (as a source of T7 RNA polymerase) was codelivered with T7-CAT (plasmid or PCR fragment). Four hours following the transfection, the medium was replaced with normal growth medium, and cells were cultured for 2 days before CAT assays were performed. For the time course study, cells were collected daily for 9 days. Cells were split $1: 1$ once on day 4 when they were $100 \%$ confluent.

\section{CAT assay}

Following the transfection, cells were washed once with PBS and lysed with $200 \mu \mathrm{l}$ of lysis buffer consisting of $0.1 \%$ Triton $\mathrm{X}-100$ in $200 \mathrm{~mm}$ Tris- $\mathrm{HCl}$ ( $\mathrm{pH} \mathrm{7.8).} \mathrm{CAT}$ assay was performed according to the method of Sankaran $^{20}$ with a few modifications. We used $1 \mathrm{~mm}$ chloramphenicol, $0.1 \mathrm{~mm}$ acetyl-CoA, and $0.1 \mu \mathrm{Ci}{ }^{3} \mathrm{H}$ acetyl-CoA for the assay mixture. The reaction was performed at $37^{\circ} \mathrm{C}$ for $1 \mathrm{~h}$, followed by extraction with $1 \mathrm{ml}$ toluene. One half of the organic phase was mixed with $3 \mathrm{ml}$ BetaMax and counted for radioactivity. One unit was defined as the amount of enzyme converting $1 \mathrm{nmol}$ of acetyl groups to chloramphenicol per min under the above reaction conditions.

\section{In vitro transcription}

In vitro transcription was performed according to the manufacturer (New England Biolabs). As the template, 
0.27 pmol of T7-CAT (fragment or plasmid) alone or complexed with either DC-chol:DOPE liposomes (1 $\mu \mathrm{g}$ DNA/10 nmol liposomes) or polylysine (1 $\mu \mathrm{g}$ DNA/0.75 $\mu \mathrm{g}$ polylysine) was used. $\alpha-{ }^{32} \mathrm{P}-\mathrm{UTP}$ along with $0.1 \mathrm{nmol}$ of NTP mix was used for transcription. Following incubation at $37^{\circ} \mathrm{C}$ for $1.5 \mathrm{~h}, 10$ units of DNaseI was added and the mixture was further incubated at $37^{\circ} \mathrm{C}$ for $20 \mathrm{~min}$. The transcribed RNA was then electrophoresed on agarose gel and visualized on the phosphor imager.

\section{Acknowledgements}

This work was supported by a grant from RGene Therapeutics Inc. We thank Dr JP Yang for making available the pCMV/T7-T7pol plasmid.

\section{References}

1 Anderson WF. Human gene therapy. Science 1992; 256: 808-813.

2 Gao X, Huang L. Cationic liposome-mediated gene transfer. Gene Therapy 1995; 2: 710-722.

3 Wicks IP et al. Bacterial lipopolysaccharide copurifies with plasmid DNA: implication for animal models and human gene therapy. Hum Gene Ther 1995; 6: 317-323.

4 Raetz CRH. Biochemistry of endotoxin. Annu Rev Biochem 1990; 59: $129-170$.

5 Redl H, Bahrami S, Schlag G, Traber DL. Clinical detection of LPS and animal models of endotoxaemia. Immunobiology 1993; 187: $330-345$.

6 Engelhardt R, Mackensen A, Galanos C. Phase I trial of intravenously administered endotoxin (Salmonella abortus equii) in cancer patients. Cancer Res 1991; 51: 2524-2530.

7 Folger KR, Wong EA, Wahl G, Capecchi MR. Patterns of integration of DNA microinjected into cultured mammalian cells: evidence for homologous recombination between injected plasmid DNA molecules. Mol Cell Biol 1982; 2: 1372-1387.

8 Manthorpe $\mathrm{M}$ et al. Gene therapy by intramuscular injection of plasmid DNA: studies on firefly luciferase gene expression in mice. Hum Gene Ther 1993; 4: 419-431.

9 Gao X, Huang L. Cytoplasmic expression of a reporter gene by co-delivery of T7 RNA polymerase and T7 promoter sequence with cationic liposomes. Nucleic Acids Res 1993; 21: 2867-2872.

10 Chen X, Li Y, Xiong K, Wagner TE. A self-initiating eukaryotic transient gene expression system based on cotransfection of bacteriophage T7 RNA polymerase and DNA vectors containing a T7 autogene. Nucleic Acids Res 1994; 22: 2114-2120.

11 Dunn JJ et al. Targeting bacteriophage T7 RNA polymerase to mammalian cell nucleus. Gene 1988; 68: 259-266.

12 Gao X, Jaffurs D, Robbins PD, Huang L. A sustained, cytoplasmic transgene expression system delivered by cationic liposomes. Biochem Biophys Res Commun 1994; 200: 1201-1206.

13 Zabner $\mathrm{J}$ et al. Cellular and molecular barriers to gene transfer by a cationic lipid. J Biol Chem 1995; 270: 18997-19007.

14 Lundberg KS et al. High-fidelity amplification using a thermostable DNA polymerase isolated from Pyrococcus furiosus. Gene 1991; 108: 1-6.

15 Gao X, Huang L. A novel cationic liposome reagent for efficient transfection of mammalian cells. Biochem Biophys Res Commun 1991; 179: 280-285.

16 Elroy-Stein O, Fuerst TR, Moss B. Cap-independent translation of mRNA conferred by encephalomyocarditis virus $5^{\prime}$ sequence improves the performance of the vaccinia virus/bacteriophage T7 hybrid expression system. Proc Natl Acad Sci USA 1989; 86: 6126-6130.

17 Farhood H, Serbina N, Huang L. The role of dioleoylphosphatidyl-ethanolamine in cationic liposome mediated gene transfer. Biochim Biophys Acta 1995; 1235: 289-295.

18 Sambrook J, Fritsch EF, Maniatis T. Molecular Cloning: A Laboratory Manual. Cold Spring Harbor Laboratory Press: Cold Spring Harbor, 1989.

19 Lee RJ, Huang L. Folate-targeted, anionic liposome-entrapped polylysine-condensed DNA for tumor cell-specific gene transfer. I Biol Chem 1996; 271: 8481-8487.

20 Sankaran L. A simple quantitative assay for chloramphenicol acetyltransferase by direct extraction of the labeled product into scintillation cocktail. Anal Biochem 1992; 200, 180-186. 